\title{
Gestión del riesgo como un instrumento para prever los estragos de las sequías y de las inundaciones en México*
}

\author{
Risk Management as an Instrument to Predict \\ the Damages of Droughts and Floods in Mexico
}

\section{Pedro César Cantú-Martínez**}

Recibido: 2016-06-05 // Aprobado: 2016-08-12// Disponible en linea: 2017-01-30

Cómo citar este artículo: Cantú-Martínez, P. C. (2017). Gestión del riesgo como un instrumento para prever los estragos de las sequías y de las inundaciones en México. Ambiente y Desarrollo, 21(40), 27-42. https://doi.org/10.11144/Javeriana.ayd21-40.grip

doi:10.11144/Javeriana.ayd21-40.grip

\section{Resumen}

El presente documento examina el contexto del recurso hídrico en México, plantea su escenario general, como también las contingencias que envuelve la sustentabilidad de este recurso natural, es el caso de las sequías y las inundaciones, las cuales son manifestaciones naturales de carácter extremo. Se bosqueja el empleo de la gestión del riesgo como un instrumento para afrontar la multitud de estragos desde la perspectiva de la vulnerabilidad.

Palabras clave: sustentabilidad; gestión del riesgo; sequías; inundaciones; agua

* $\quad$ Este artículo de reflexión presenta de manera crítica y analítica los resultados de investigaciones sobre el manejo del recurso hídrico en México, en especial sobre las sequías y las inundaciones y la gestión del riesgo como instrumento para evitar los estragos que estos fenómenos conllevan.

** Ph. D. en Ciencias Biológicas, profesor e investigador de la Universidad Autónoma de Nuevo León, México, Facultad de Ciencias Biológicas, San Nicolás de los Garza, N. L., México. Correos electrónicos: cantup@ hotmail.com; pedro.cantum@uanl.mx 


\begin{abstract}
This article examines the context of the water resource in Mexico, presenting its general scenario as well as the hazards around the sustainability of this natural resource-such as droughts and floods- which are extreme natural events. The use of risk management is outlined as an instrument to deal with the many damages from the perspective of vulnerability.
\end{abstract}

Keywords: sustainability; risk management; droughts; floods; water 


\section{Introducción}

Se reconoce que el agua es particularmente importante para la vida de todo ser humano como de todo colectivo social. No obstante, de acuerdo con la Oficina del Alto Comisionado de las Naciones Unidas para los Derechos Humanos (2011, p. 1), aún coexisten "884 millones de personas carecen de acceso a fuentes mejoradas de agua potable, y 2.500 millones no disponen de servicios mejorados de saneamiento".

Según Cantú-Martínez (2012, p. 21), el recurso hídrico, particularmente el agua dulce, se ha constituido en un recurso muy apreciado, "ya que su disponibilidad en el mundo se calcula de 9,000 a 14,000 km cúbicos, de los cuales sólo 4,200 km cúbicos son viables para ser utilizados por nuestra sociedad", y este volumen con el que contamos no varía. Entre las principales razones de la actual situación y que afectan este recurso natural tan apreciado encontramos la "pobreza, las desigualdades y la disparidad en las relaciones de poder, y se ven agravadas por los retos sociales y ambientales, como la urbanización cada vez más rápida, el cambio climático, y la creciente contaminación y merma de los recursos hídricos" (Oficina del Alto Comisionado de las Naciones Unidas para los Derechos Humanos 2011, p. 1).

Por lo tanto, el agua es fundamental para proveer una mejor calidad de vida a los seres humanos, lo que se traduce en bienestar y se convierte en un recurso trascendental que repercute claramente en la vida social de toda comunidad. Es así como el agua se constituye como un suceso relevante en nuestras vidas, principalmente cuando se presentan precipitaciones pluviales agudas que se constituyen en acontecimientos catastróficos, esencialmente para las comunidades vulnerables que se encuentran en los centros urbanos y que abundan en el contorno de las ciudades, como también cuando se carece de la disponibilidad de este recurso y los efectos de dicha carencia se ciernen sobre la población rural y también sobre la de los grandes conglomerados poblacionales.

En lo referente al cambio climático, publicaciones recientes (Miguel Velasco et al., 2012; Ponce Cruz, Cantú-Martínez y Puente Quintanilla, 2013; Cantú-Martínez, 2014) muestran que este suceso prorrogará durante los próximos ańos sus efectos en el agua en distintas regiones del mundo. Por lo tanto, existe una aceptación general entre los académicos y la comunidad internacional de que dicho fenómeno - el cambio climático - deja secuelas en el ciclo hidrológico. Debido a esto se observa con gran interés su impacto en el recurso hídrico en el mundo, que reduce la disponibilidad tanto en cantidad como en calidad (Peña Ramírez, 2012), lo que de acuerdo con Cantú-Martínez (2014, p. 34) se puede observar "a través de la alteración del régimen de lluvias, fenómenos con una abundancia de precipitaciones pluviales en ciertos sitios que en intervalos cortos de tiempo generan inundaciones $y$, por otra parte, ciclos extendidos de tiempo con severas sequías".

En el presente manuscrito se explora en forma sucinta el estado de los recursos hídricos en México y se abordan las eventualidades que implican las sequías y las inundaciones, que son fenómenos naturales extremos, y se plantea la utilidad de la gestión del riesgo como una herramienta para evitar multitud de estragos en México. Como sostiene Angelotti Pasteur (2014, p. 71), "entre los estudiosos que abordan problemáticas sociales relacionadas con los desastres provocados por fenómenos naturales o antrópicos, existe común acuerdo en que éstos no son eventos súbitos inesperados". Lo anterior conlleva que la gestión del riesgo sea considerada un área de creciente interés donde se analizan situaciones complicadas y dinámicas (Moreno Plata, 2011).

\section{Disponibilidad del recurso agua en México}

México, en comparación con otros países, de acuerdo con el Grupo Achipáhuac (2008, p. 62), cuenta con escenarios "relativamente favorables en términos de disponibilidad de agua subterránea y superficial, así como una precipitación pluvial y grados de humedad relativa en el ambiente bastante 
aceptables". No obstante, subsisten diferencias muy evidentes en la distribución espacial del recurso hídrico. Por ejemplo, la precipitación pluvial en México, según diferentes organismos como el Fondo para la Comunicación y la Educación Ambiental, el Centro Mexicano de Derecho Ambiental y la Presencia Ciudadana Mexicana (2006), asciende a un volumen aproximado de $1511 \mathrm{~km}^{3}$ por ańo. Debido a ello México se cataloga como un país con baja disponibilidad de agua.

Del volumen mencionado, cerca del $50 \%$ corresponde a los estados del sur y del sureste, mientras que en la zona norte, cuyas características climáticas favorecen un ambiente semiárido y árido, solo se presenta un $25 \%$ de estas precipitaciones, tan solo cuatro meses del año. Esta es una de las particularidades que se unen a las eventualidades de insuficiencia que tienen que ver con la gran variedad de regiones y climas del país. Esto se comprueba mediante las estadísticas de precipitación anual del periodo de 1971 al 2000. En estas la Comisión Nacional de Agua (2013) registra que la región hidrológica administrativa de la Frontera Sur acogió un volumen promedio de $1846 \mathrm{~mm}$, mientras que la región de la Península de Baja California apenas llegó a los $169 \mathrm{~mm}$ en promedio anual.

Datos estadísticos de la misma entidad (Comisión Nacional del Agua, 2007) revelan que en el estado de Baja California la incidencia de precipitación pluvial es muy exigua, únicamente $202 \mathrm{~mm}$ de lluvia al año en promedio, mientras que en la entidad federativa de Tabasco, ubicada al sureste de la nación, la magnitud de la precipitación es doce veces más abundante y alcanza valores promedio anuales de $2410 \mathrm{~mm}$. Por otra parte, la Secretaría de Medio Ambiente y Recursos Naturales (2013a) reporta que en el 2012 Baja California Sur consignó, aproximadamente, $70 \mathrm{~mm}$ de precipitación, mientras que en las entidades federativas de Chiapas y Tabasco el promedio anual de lluvia alcanzó valores de 2373 y 2478 mm, respectivamente. Además, documenta que en el lapso 1981-2010 la precipitación promedio anual fue de $935,7 \mathrm{~mm}$ en todo el país.

En México tanto el crecimiento demográfico como el crecimiento económico se han dado preferentemente en el centro y en el norte, zona que cuenta con el $31 \%$ de la disponibilidad de agua nacional y alberga al $77 \%$ de la población, lo que contrasta diametralmente con la región sureste, que concentra el $23 \%$ de la población y cuenta con el $69 \%$ del recurso hídrico aprovechable (Monforte García y Cantú-Martínez, 2009). Carabias y Landa (2005) sostienen que el consumo de agua per cápita en las naciones desarrolladas fluctúa entre 500 y 800 litros diarios, mientras que en las naciones en desarrollo oscila entre 60 y 150 litros diarios. Esto entraña una correspondencia entre el crecimiento de los complejos urbanos, el desarrollo de las regiones y la demanda de consumo de agua.

En México las demandas de consumo son muy variadas. Hay una presión constante sobre el recurso hídrico, no solo por las actividades económicas, sino también por el apremio que existe en las zonas urbanas por abastecerse de agua para una población cada vez más mayor, por lo cual la gestión de dicho recurso constituye uno de los más grandes retos en el presente, esencialmente por la variabilidad espacial y temporal de su disponibilidad en el país (Tiburcio Sánchez y Perevochtchikova, 2012).

La demanda de agua por habitante es muy desigual, lo cual obedece a diferentes situaciones como las actividades socioeconómicas, las condiciones climatológicas y la disposición del recurso hídrico, entre otros factores. El Fondo para la Comunicación y la Educación Ambiental, el Centro Mexicano de Derecho Ambiental y Presencia Ciudadana Mexicana (2006) cuantifican los siguientes consumos:

1) La dotación promedio en el medio urbano en el país es de 250 litros/habitante/día y en general, por fugas de diferente tipo y origen, se pierden en los sistemas cerca de 100 lts./hab./ día, lo que hace que el consumo promedio por habitante sea de 150 lts./día. Estos son algunos ejemplos en lugares de México (exclusivamente de uso doméstico): Tijuana, B.C.: 176 lts./hab./ día; León, Gto.: 116 lts./hab./día; Monterrey, N. L.: 180 lts./hab./día; Mexicali, B. C.: 220 lts./ hab./día; Naucalpan, Estado de México: 225 lts./hab./día. 
2) En el medio rural, donde hay un sistema formal de abasto, la dotación promedio es de 150 lts./hab./día y los consumos reales son en promedio de 100 lts./hab./día. Generalmente estos consumos en el medio rural se incrementan por el riego de hortalizas y el abrevadero para ganado. Estos consumos de agua se distribuyen en regadera, lavado de ropa, sanitarios, alimentos, etc. (p. 40)

Por otra parte, en el 2010 Pineda Pablos, Salazar Adams y Buenfil Rodríguez señalaron que el consumo neto per cápita para Tijuana pasó a ser de 155 litros por habitantes por día, con una eficiencia física del $81 \%$; en León 117 litros por habitantes por día y una eficiencia física del $57 \%$; en Monterrey 193 litros por habitantes por día y una eficiencia física del $70 \%$, y por último, en Mexicali 270 litros por habitantes por día y una eficiencia física del $83 \%$.

Lo anterior ha planteado distintos problemas para la conservación del agua, sobre todo si se considera que en México, de acuerdo con Carabias y Landa (2005), "Los 653 acuíferos del país proveen $70 \%$ del volumen de agua que se suministra a las ciudades para abastecer a casi 75 millones de personas, así como para regar una tercera parte de la superficie agrícola irrigada del país" (p. 59). Esta explotación ha provocado que el $15.6 \%$ de estos mantos acuíferos se encuentren sobreexplotados, lo que ha conllevado que la provisión de agua subterránea de México decline en el orden de $6 \mathrm{~km}^{3}$ por año (Comisión Nacional del Agua, 2004). Esta reducción ocasiona que en algunas regiones del país se busquen fuentes de aprovisionamiento de agua que garanticen el abastecimiento de los grandes núcleos poblacionales que albergan, como sucede en la zona del valle de la Ciudad de México y en el Área Metropolitana de Monterrey.

De forma muy particular, "la ciudad de México se ha transformado en todo un paradigma nacional y tal vez mundial, que impacta negativamente la cuenca donde se asienta" (Grupo Achipáhuac, 2008, p. 63), por los altos volúmenes de agua, de carácter urbano e industrial que demanda. Peńa Ramírez y el Grupo Achipáhuac (2008) describen esto, lacónicamente, como una "concentración acelerada y, efectivamente, desordenada, que chupa el agua de su entorno, la consume de forma minera y la contamina" (p. 118).

Por su parte, Moreno Vázquez, Marañón Pimentel y López Córdova (2010) afirman que los "casos críticos se presentan en estados del centro y norte de la República Mexicana, en particular en la cuenca del río Lerma (Guanajuato y Querétaro), en la región de La Laguna (Coahuila-Durango), en la península de Baja California, y en Aguascalientes, Chihuahua y Sonora" (p. 80). Esto ha demostrado, como sostiene Velázquez Simental (2010), que se "ha disminuido la disponibilidad de agua per cápita de 17,825 millones de metros cúbicos en 1950 a 4,097 millones de metros cúbicos en 2010 ” (p. 5).

No obstante que nuestro país cuenta con una ubicación geográfica bastante favorecida, por contar con una combinación de climas templados y tropicales que le suministran condiciones adecuadas para una muy buena disponibilidad de agua natural, como señalan Carabias y Landa (2005), persisten problemas coyunturales que atañen a distintos aspectos que impiden contar con este beneficio potencial. Entre estos mencionan:

- Déficit de conocimiento y falta de recursos humanos capacitados en geociencias.

- Falta de grupos de investigación interdisciplinarios para la administración de acuíferos.

- Gran dependencia tecnológica.

- Pocos acuíferos instrumentados.

- Falta de uniformidad metodológica en el diagnóstico y el monitoreo de la calidad del agua subterránea.

- Falta de control de las extracciones y oposición a la medición. 
- Falta de visión de largo plazo en el sector productivo.

- Ausencia de políticas que favorezcan el cuidado de los acuíferos.

- Falta de capacitación y conocimiento en el manejo y uso de sistemas subterráneos en los consejos de cuenca.

- Incompatibilidad en la gestión entre algunos consejos de cuenca y las Cotas.

- Regulación que no incentiva el ahorro.

- Insuficientes mecanismos para el ordenamiento de acuíferos.

- Desinformación sobre criterios, mecanismos y alcances de las vedas. (p. 67)

En México, en el terreno de la gestión urbana del agua, esta ha sido de suma importancia e interés nacional, particularmente porque se reconoce, de acuerdo con Rolland y Vega Cárdenas (2010), que "la desigualdad en la repartición de este recurso en el territorio, su contaminación y la falta de mantenimiento de las infraestructuras hidráulicas son responsables de su escasez" (p. 155).

A lo anterior habría que añadir que la gestión del agua en las zonas urbanas se caracteriza por la incorrecta operación de los sistemas de distribución, donde existen serias dificultades financieras para rehabilitar y mantener el funcionamiento adecuado de la infraestructura existente para el acopio y la distribución del agua, como también deficientes prácticas de consumo en los usuarios y falta de conocimiento sobre reuso, separación y aprovechamiento del agua proveniente de las precipitaciones pluviales (Fondo para la Comunicación y la Educación Ambiental, Centro Mexicano de Derecho Ambiental y Presencia Ciudadana Mexicana, 2006). Lo anterior describe un escenario poco alentador para la población del país. A lo que Barkin (2006) agrega:

El sistema de gestión hídrica en México está en crisis. Aunque las autoridades nos aseguran que más del $90 \%$ de la población tiene acceso al agua potable y que una parte un poco menor tiene conexiones al alcantarillado, la realidad es que el país está sufriendo grandes estragos por su inadecuada disponibilidad en calidad y cantidad. (p. 3)

En cuanto a la evolución de la población que habita en grandes ciudades, preferentemente superiores a los 100000 habitantes, se ha venido observando que la cobertura de agua potable se lleva a cabo más rápidamente que en centros poblacionales más pequeños.

La Secretaría de Medio Ambiente y Recursos Naturales (2013a) señala que el volumen de abastecimiento público nacional asciende a $11 \mathrm{~km}^{3}$, por lo cual se ha tenido que construir un gran conjunto de infraestructura hidráulica de presas en todo el territorio nacional para garantizar este suministro. En la actualidad este conjunto de obras representa en número 4462 presas, de las cuales 667 son catalogadas como grandes, y su capacidad total de almacenamiento es de alrededor de $150 \mathrm{~km}^{3}$ para todo el complejo de instalaciones hidráulicas. En el 2010, por ejemplo, el volumen almacenado de agua alcanzó el $91 \%$, mientras que en 1996, considerado escaso en precipitaciones pluviales, se logró tan solo el $71 \%$.

Por otra parte, a pesar que la Ley de Aguas Nacionales (2013) eleva en el contexto legal nacional al agua como un bien público federal con valor socioeconómico y ambiental, y además conmina a los usuarios a dar tratamiento al recurso hídrico que se emplee, con la finalidad de habilitar su uso subsecuente en otras actividades o aplicaciones, conservar el equilibrio y la calidad de los sistemas naturales que las proveen, dar cumplimiento a las normas oficiales mexicanas o las condiciones particulares de descarga, esto no se realiza a cabalidad en el territorio nacional, debido a que "las ciudades e industrias tratan sólo parcialmente sus efluentes y el agua cruda contamina los mantos freáticos" (Grupo Achipáhuac, 2008, p. 64). 
Lo antes mencionado resulta evidente ya que de las redes fluviales y cuerpos de aguas nacionales que monitorea la Comisión Nacional del Agua, el 74\% muestra diferentes niveles de contaminación, pues contiene agentes extraños que han modificado su composición física, química y por ende su calidad, en particular en las cuencas del Lerma, Alto Balsas, Río Colorado y Alto Pánuco, las cuales cuentan con mayor cantidad de afluentes con un alto grado de contaminación (Fondo para la Comunicación y la Educación Ambiental, Centro Mexicano de Derecho Ambiental y Presencia Ciudadana Mexicana, 2006), y esto atenta contra la sustentabilidad en materia de recursos hídricos. A lo anterior Tiburcio Sánchez y Perevochtchikova (2012) agregan que "Las soluciones implementadas hasta ahora [en México] han sido de tipo reactivo, temporal y fragmentado" (p. 156).

\section{Situaciones naturales extremas en relación con el recurso agua}

Las situaciones naturales extremas relacionadas con sequías e inundaciones son denominadas por Aplízar Marín (2009) amenazas socionaturales, las cuales entiende como "El fenómeno de una mayor ocurrencia de eventos relativos a ciertas amenazas geofísicas e hidrometeorológicas, tales como aludes, inundaciones, subsidencia de la tierra y sequías, que surgen de la interacción de las amenazas naturales con los suelos y los recursos ambientales explotados en exceso o degradados" (p. 2). Aquí se examina dicha situación en México.

\section{Las sequías}

La sequía es un acontecimiento extraordinario de la naturaleza cuyos indeseables efectos se sufren mayormente donde el agua escasea, pero además se acentúa en colectividades que no se encuentran preparadas para ello. De acuerdo con Marcos Valiente (2001), esta puede considerarse "la deficiencia de precipitaciones durante un período de tiempo prolongado" (p. 60); sin embargo, también afirma que puede ser catalogada como meteorológica, agrícola, hidrológica y socioeconómica.

En una aproximación sucinta, estas diferentes sequías se distinguen por lo siguiente: 1) meteorológica: se identificada por la carencia de lluvias en lapsos cortos; 2) agrícola: se particulariza por una humedad escasa en el suelo, y es de carácter estacional, lo cual provoca bajos rendimientos agrícolas; 3) hidrológica: es reconocida por los amplios lapsos que permanece — varios años- y es perceptible debido a la disminución de las corrientes de ríos, o bien por la disminución en los niveles de almacenamiento de presas y acuíferos; 4) socioeconómica: es aquella que se identifica porque sus estragos se ciernen sobre las actividades productivas, cuyos procesos contemplan como insumo principal el recurso hídrico (Velasco, Ochoa y Gutiérrez, 2005).

La sequía ha cobrado gran notoriedad por los perjuicios y estragos que ocasiona, los cuales con mucha frecuencia son de gran magnitud. Incluso, en ocasiones son mayores a los que se generan por otros fenómenos hidrometeorológicos. No obstante que en la parte sur y sureste del país se presentan lluvias copiosas, hay zonas donde no se registran en las proporciones necesarias, como es el caso de las entidades federativas ubicadas en el norte y en el centro del país, entre ellas Durango, Baja California, Chihuahua, Coahuila, Nuevo León, San Luis Potosí, Aguascalientes y Zacatecas (García Jiménez, Fuentes Mariles y Matías Ramírez, 2002; Comisión Intersecretarial de Cambio Climático, 2012).

En México se tiene documentado, de acuerdo con Castorena et al. (1980), que ya se daba aún antes de la Conquista. Contreras Servín (2005) asevera, por su parte, que en el siglo XIX se pueden consignar cinco notables sequías que aquejaron al país en los lapsos de 1808-1811, 1868, 1877, 18841885 y 1892-1896. Por otra parte, el Centro Nacional de Prevención de Desastres (2001) advierte que durante el siglo XX hubo cinco periodos de sequía: 1948-1954, 1960-1964, 1970-1978, 1993-1996 y 1998. Al inicio del siglo XXI sobrevinieron inclementes lapsos de sequía en 2000-2003, 2009 y 20112012 (Secretaría de Medio Ambiente y Recursos Naturales, 2013a). 
Según García Jiménez, Fuentes Mariles y Matías Ramírez (2002), "Los efectos de una sequía se dejan sentir en el aspecto económico y social, ya que las pérdidas en cosechas, animales, disminución de la producción industrial, y otros, ocasionan la reducción del poder adquisitivo de la población" (p. 9); sin embargo, también deben considerarse como efectos dependientes cuyas manifestaciones deterioran el entorno natural, los incendios forestales y el incremento de la erosión de los suelos.

Por esa razón, las pautas de prevención para enfrentar las sequías residen, en primera instancia, en políticas del empleo eficaz del recurso hídrico en los domicilios, en las actividades agrícolas y ganaderas, así como también en la industria. En segunda instancia, el propósito está enfocado en ampliar la construcción de infraestructura hidráulica dedicada al almacenamiento y la distribución del agua (García Jiménez, Fuentes Mariles y Matías Ramírez, 2002). Sin embargo, de acuerdo con la Secretaría de Medio Ambiente y Recursos Naturales (2013b), en México para aumentar la resiliencia a las sequías es necesario:

\author{
-Desarrollar planes de contingencia ante las sequias, que asignen responsabilidades concretas \\ y compromisos a los actores, como usuarios y dependencias. \\ -Minimizar las pérdidas en redes de agua potable. \\ -Promover el uso eficiente del agua en las ciudades. \\ -Fomentar e incentivar el reúso del agua. \\ -Controlar la sobreexplotación de los acuíferos y monitorear el comportamiento de los mismos. \\ -Promover el uso de cultivos de baja demanda. \\ -Incentivar la aplicación de técnicas avanzadas de riego. \\ -Impulsar la captación de agua de lluvia, especialmente en la Ciudad de México. (p. 13)
}

En otros términos, Martínez Austria (2001) y Velasco, Ochoa y Gutiérrez (2005) consideran pertinente - reconociendo que la problemática es de carácter nacional— que en México las políticas públicas contemplen acciones primordiales para la gestión del agua y la posibilidad de aproximarse al desarrollo sustentable en circunstancias como las que se han mencionado. Estas acciones serían: 1) que todos los actores implicados en la gestión del recurso hídrico sean considerados en la toma de decisiones; 2) incentivar el pago de tarifas que cubran el costo de los servicios, acompañado de una política de subsidios que favorezca a los más desamparados; 3) incrementar la capacidad tecnológica para mejorar el diseño, la operación y el mantenimiento de los sistemas proveedores y de almacenamiento de agua; 4) incentivar los acuerdos internacionales para el manejo integral de cuencas hidrológicas internacionales y 5) contar con suficientes recursos económicos e instrumentos financieros para sostener los programas institucionales que el sector hidrológico demanda.

\title{
Inundaciones
}

Según la Secretaría de Medio Ambiente y Recursos Naturales (2013b), México tiene una fisiografía y características climáticas que le dan unas condiciones orográficas e hidrológicas las cuales favorecen la presencia de fenómenos hidrometeorológicos como las inundaciones, las cuales han ocurrido desde tiempos muy antiguos (Audefroy, 2012). Según el Centro Nacional de Prevención de Desastres (Salas Salinas y Jiménez Espinoza 2004), se considera una inundación:

[...] aquel evento que debido a la precipitación, oleaje, marea de tormenta, o falla de alguna estructura hidráulica provoca un incremento en el nivel de la superficie libre del agua de los ríos o el mar mismo, generando invasión o penetración de agua en sitios donde usualmente no la hay y, generalmente, daños en la población, agricultura, ganadería e infraestructura. (p. 5) 
Lo anterior describe un escenario de alta vulnerabilidad a inundaciones en muchas regiones del país, por eventos hidrometeorológicos extremos, como es a través de la presencia de lluvias con carácter torrencial, ventarrones y ciclones, lo que se exacerba en lugares donde existe un deterioro sensible del entorno, con manifestaciones de erosión, remoción de la masa boscosa y también intensas modificaciones del uso de suelo en las cuencas por la urbanización.

Lo referido en el acápite precedente altera la capacidad hidrológica de las cuencas, aumenta la probabilidad de que ocurran inundaciones y afecta su magnitud. Dichas inundaciones posteriormente se manifiestan en eventos de azolve en las represas que perturban el potencial de producción de energía hidroeléctrica y la calidad del agua para consumo doméstico e industrial. Además causan daños a la infraestructura urbana y vial, lo que conllevará un alto costo económico y social, con pérdida de vidas humanas, en especial en aquellas comunidades asentadas a las orillas de los cauces, así como en las faldas de los cerros, ya que es factible que haya derrumbes y deslizamientos por los elevados grados de humedad que persisten en el suelo en esos días (Casillas González, 2004; Cantú-Martínez, 2014). Por consiguiente, en muchas ocasiones la respuesta de las autoridades y de la población es tardía, aun cuando muy comprometida.

Para mitigar los daños por inundaciones, de acuerdo con el Centro Nacional de Prevención de Desastres (Salas Salinas y Jiménez Espinoza, 2004):

[...] es indispensable emprender acciones de protección. Éstas pueden ser de dos tipos: medidas estructurales (construcción de obras), o medidas no estructurales (indirectas o institucionales). Por otra parte, entre las medidas no estructurales se encuentran aquéllas cuya finalidad es informar oportunamente a las poblaciones ribereńas de la ocurrencia de una posible avenida, para que no haya muertes y se minimicen los dańos. En este rubro se incluyen los reglamentos de usos del suelo, el alertamiento y los programas de comunicación social y de difusión. (p. 27)

No obstante, considerado los aprendizajes que han dejado las inundaciones ocurridas en el país en distintos lugares, los esfuerzos de las instituciones públicas deberían encaminarse a: 1) poseer un sistema avanzado de predicción hidrológica; 2) emplear y respetar los planes de ordenamiento territorial, para evitar la alteración antropogénica de los sistemas hidrológicos; 3) contar con un programa permanente de conservación y rehabilitación del entorno natural; 4) estimular el desarrollo de las capacidades institucionales, tecnológicas y de formación especializada y 5) mejorar la coordinación interinstitucional entre los tres niveles de gobierno como son el municipal, el estatal y el federal.

\section{Gestión del riesgo como instrumento de prevención}

Los procedimientos de gestión, particularmente observados como instrumentos para la mejora continua, nos han habilitado como sociedad para advertir un escenario holístico de carácter medioambiental, económico y social, con representación prospectiva, que nos aproxima al desarrollo sustentable. Dichos procedimientos contemplan una noción, un conocimiento y una praxis en pleno progreso.

La gestión del riesgo como una herramienta para la sustentabilidad se precisa como el procedimiento que estudia y pondera los efectos y las consecuencias de los desastres naturales o antropogénicos y contempla operaciones de implementación con carácter anticipatorio y correctivo. Asimismo, el término gestión del riesgo involucra "tres políticas públicas distintas: la identificación del riesgo (percepción individual, representación social y estimación objetiva), la reducción del riesgo (prevención-mitigación) y el manejo de desastres (respuesta y recuperación)" (Álvarez Gordillo et al., 2008, p. 924).

De acuerdo con Narváez, Lavell y Pérez Ortega (2009), el riesgo puede considerarse una "condición latente que, al no ser modificada o mitigada a través de la intervención humana o por medio de un cambio en las condiciones del entorno físico-ambiental, anuncia un determinado nivel de impacto 
social y económico hacia el futuro" (p. 9). En esta condición latente se reconoce que el riesgo es producto de la relación de dos condiciones preexistentes: la amenaza y la vulnerabilidad. La amenaza trazada por los fenómenos naturales o actividades antropogénicas y la vulnerabilidad representada por los grados exposición y fragilidad del sistema receptor de estos eventos.

Si bien la amenaza no es controlable, particularmente en los sucesos naturales, como en este caso las sequías y las inundaciones, sí se puede moderar la vulnerabilidad al realizar acciones que reduzcan la intensidad de los sucesos planteados por la amenaza y de tal suerte aminorar los daños socioeconómicos y ambientales (véase figura 1). Por esta razón, Keipi, Mora Castro y Bastidas (s. f.) sostienen que en la gestión del riesgo deben considerarse dos etapas: la ex-ante y la ex-post, lo que contempla elementos claves que considerar, como son: "i) La identificación y análisis del riesgo, ii) la concepción y aplicación de medidas de prevención y mitigación, iii) la protección financiera mediante la transferencia o retención del riesgo y iv) los preparativos y acciones para las fases posteriores de atención, rehabilitación y reconstrucción" (p. 8).

En lo que sigue se pone el énfasis sobre las consideraciones ex-ante, que pueden denominarse predesastre, ya que al evaluar los estragos producidos por las sequías e inundaciones en México, y al no aprovecharse el recurso hídrico adecuadamente, pareciera que dichas sequías e inundaciones se han acentuado en intensidad y recurrencia, pero esto más bien obedece a que se ha acrecentado la vulnerabilidad del entorno socioambiental en las unidades geográfico-espaciales que las padecen, y se muestra que cada vez se requiere una menor intensidad en las amenazas para que los umbrales de perjuicios socioeconómicos y de víctimas aumenten. En este sentido, Romero y Maskrey (1993) señalan tres aspectos que aumentan la vulnerabilidad en la sociedad:

1) Cuando la gente ha ido poblando terrenos que no son buenos para vivienda, por el tipo de suelo, por su ubicación inconveniente con respecto a huaycos [desprendimientos de lodo y rocas], avalanchas, deslizamientos, inundaciones, etc.

2) Cuando ha construido casas muy precarias, sin buenas bases o cimientos, de material inapropiado para la zona, que no tienen la resistencia adecuada, etc.

3) Cuando no existe condiciones económicas que permitan satisfacer las necesidades humanas (dentro de las cuales debe contemplarse la creación de un hábitat adecuado). (p. 8)

\section{Gestión del riesgo}

Gestión del riesgo

Análisis del riesgo

Medidas de prevención y mitigación

Medidas de regulación

\section{Gestión del riesgo}

Preparativos para la atención

Acciones de rehabilitación

Actividades de reconstrucción

\section{Protección financiera Comunicación del riesgo}

Figura 1. Gestión del riesgo 
Álvarez Gordillo et al. (2008) añaden a lo anterior que esta vulnerabilidad se incrementa también cuando "una sociedad que, por su desarrollo en infraestructura, productivo, territorial, institucional, cultural, político, social, ambiental e histórico resulte incapacitada para prepararse, enfrentar o recuperarse de los impactos de los eventos físicos externos" (pp. 921-922). Si no fuera este el contexto, y se contara con los recursos técnicos, económicos y de organización necesarios, pero sin claridad teórica para reconocer qué tipo de acciones llevar a cabo en caso de una amenaza natural inminente, esto también promovería la vulnerabilidad, evidenciando la falta de congruencia entre el empleo de los recursos y las actividades que es preciso realizar. En tal caso las acciones se concentran durante las emergencias, esto es, postdesastre (Wilches-Chaux, 1993).

De acuerdo con lo anterior, los escenarios de vulnerabilidad se van creando y ańadiendo gradualmente y van conformando un entorno de riesgo que en muchas ocasiones pasa inadvertido y cuando se observa se desatiende o minimiza por parte de las autoridades, y aun de la sociedad misma. En otras palabras, la vulnerabilidad la construye la sociedad paulatinamente en el tiempo.

Según Chuquisengo y Gamarra (citados por Alpízar Marín, 2009), "la gestión del riesgo es un proceso planificado, concertado, participativo e integral de reducción de las condiciones de riesgo de desastres, en la búsqueda del desarrollo sostenible" (p. 21). Desde entonces, dicho marco referencial ha constituido un real adelanto en el concierto internacional que permite apreciar el riesgo de forma sistémica y holística, donde los distintos actores sociales participan activamente, realizándolo, en esencia, en el ámbito local, que incide en la corrección o alteración de los escenarios que gestan el riesgo y en la regulación de los factores que los magnifican (Alpízar Marín, 2009).

Para elaborar este constructo socioambiental, particularmente en la etapa predesastre, como una respuesta conexa a la realidad en que subiste la colectividad social afectada, es conveniente entender la gestión del riesgo como una aplicación social de carácter interdisciplinar e interistitucional, con una responsabilidad compartida y un enfoque el cual asume que el riesgo es una oportunidad para el desarrollo sustentable que involucra procesos de largo plazo, de planeación e implementación de obras, así como de control y apego a las regulaciones legales que se instituyen en el entorno fisiográfico y social, y no tan solo llevar a cabo la rehabilitación y reconstrucción de los daños causados por las amenazas naturales. Desde esta perspectiva, la gestión integral del riesgo no busca solo tener respuestas postdesastres, sino también evitar o abreviar su impacto en la sociedad, lo cual conlleva trabajar solidariamente con el único propósito de prevenir los desastres, con una visión de gestión prospectiva y sustentable (Lampis y Rubiano, 2012).

\section{Postura en México}

En México en el 2012 se expide Ley General de Protección Civil, la cual tiene como papel principal coordinar los esfuerzos de los tres órdenes de gobierno (federal, estatal y municipal) en materia de protección civil, para llevar a cabo la gestión integral de riesgos. Esta gestión contempla, de acuerdo con la ley en mención:

El conjunto de acciones encaminadas a la identificación, análisis, evaluación, control y reducción de los riesgos, considerándolos por su origen multifactorial y en un proceso permanente de construcción, que involucra a los tres niveles de gobierno, así como a los sectores de la sociedad, lo que facilita la realización de acciones dirigidas a la creación e implementación de políticas públicas, estrategias y procedimientos integrados al logro de pautas de desarrollo sostenible, que combatan las causas estructurales de los desastres y fortalezcan las capacidades de resiliencia o resistencia de la sociedad. Involucra las etapas de: identificación de los riesgos y/o su proceso de formación, previsión, prevención, mitigación, preparación, auxilio, recuperación y reconstrucción. (Art. 2.o, fracción XXVII) 
La gestión integral de riesgos tiene que ver especialmente con la gestión integral anticipada, la cual se ocupa previamente de las posibles consecuencias de un agente denominado perturbador. Estas acciones abarcan:

I. Conocimiento del origen y naturaleza de los riesgos, además de los procesos de construcción social de los mismos;

II. Identificación de peligros, vulnerabilidades y riesgos, así como sus escenarios;

III. Análisis y evaluación de los posibles efectos;

IV. Revisión de controles para la mitigación del impacto;

V. Acciones y mecanismos para la prevención y mitigación de riesgos;

VI. Desarrollo de una mayor comprensión y concientización de los riesgos, y

VII. Fortalecimiento de la resiliencia de la sociedad. (Art. 10)

Sin embargo, los estudios de vulnerabilidad y riesgos sobre el cumplimiento de los numerales II y III han sido ignorados, o bien se excluyen de los planes de desarrollo urbano intencionalmente, como sostiene Toscana Aparicio (2011). Así lo muestran los acontecimientos acaecidos en las porciones bajas del estado de Tabasco, las cuales han sido destinadas para edificar nuevos desarrollos urbanos, donde se ha evidenciado que, en eventos extraordinarios de precipitaciones pluviales, los cursos de agua recuperan sus trayectos naturales arrasando con todo lo que encuentran a su paso.

Como se encuentra documentado, en octubre del hubo una serie de devastaciones en dicha entidad federativa que afectó aproximadamente a 1500 localidades. Asimismo, el $62 \%$ de la superficie del estado se encontraba cubierto de agua, dejando a "a casi 1.5 millones de damnificados $(75 \%$ de la población del estado), casi 6,500 kilómetros de carreteras y caminos afectados ( $73 \%$ de la red del estado) y 132 puentes, 570 mil hectáreas agrícolas siniestradas, 123 mil viviendas con afectaciones", según registrara la Secretaría de Gobernación en México (2008, p. 4) en su reporte.

En el caso de la sequía se evidencia que en México este fenómeno pasó de ser un evento natural a uno de génesis antrópica, inducido y exacerbado por el cambio climático, el cual es poco percibido por la población a menos que sea muy prolongado. Esto es producto de la presión existente por parte del desarrollo económico y el desmesurado incremento de la demanda del recurso hídrico por parte de los centros poblacionales, cuyos efectos se han resentido más en el norte del país, en ciudades como Hermosillo y Monterrey (Velasco, Ochoa y Gutiérrez, 2005) y recientemente en la Ciudad de México.

Ante lo expuesto precedentemente, en México se creó el Programa Especial de Cambio Climático 2009-2012, el cual es muestra del interés del gobierno federal por los problemas surgidos debido a este fenómeno global y que orientó el trabajo colectivo e intersecretarial de manera transversal, con un enfoque enteramente de prevención de riesgos durante este periodo (Comisión Intersecretarial de Cambio Climático, 2012). Este programa tuvo vigencia de agosto del 2009 a finales del 2012 y se constituyó en un instrumento nacional de política pública de gestión integral del riesgo que permitió sumar acciones para adoptar medidas y ajustes, tanto en los sistemas naturales como en los sistemas socioambientales, con la finalidad de contribuir a la disminución del volumen de población considerada vulnerable al cambio climático, donde son particularmente muy relevantes los estragos de las sequías e inundaciones.

Entre las acciones específicas en el ámbito que se aborda en el este documento se encuentra el establecimiento de cinco centros regionales de atención de emergencias ante fenómenos hidrometeorológicos extremos, como también la creación de proyectos de prevención, edificación y sostenimiento de instalaciones — como embalses—, además de servicios, plasmados en estudios y planes de 
manejo de cuencas para observar la disponibilidad de agua superficial para protección de la población, especialmente durante las sequías. Aunado a todo la anterior, se elabora un atlas nacional que documenta las variables climatológicas para disminuir la vulnerabilidad creada por el cambio climático, lo que permite integrar medidas precautorias y preventivas a los planes de desarrollo estatales y municipales que inciden en el ordenamiento territorial, de manera que se reduzca la vulnerabilidad de la población.

En la actualidad se ha dado continuidad a este programa para un periodo del año 2014 al 2018 (Comisión Intersecretarial de Cambio Climático, 2014), el cual tendrá una observancia durante el lapso del gobierno constitucional vigente. Las proyecciones que se mencionan, de acuerdo con el Programa Especial de Cambio Climático 2014-2018, son alarmantes, ya que una gran parte del territorio nacional, particularmente la parte norte, se hará más seca y las sequías se acentuarán, con el lógico incremento de la demanda de agua, sustancialmente proveniente de los grandes centros urbanos del país. En contraste, las precipitaciones se harán más intensas, lo que de acuerdo con el marco de referencia del programa pondrá en riesgo a cerca de dos millones de personas que se hallan en el país en una condición de moderada a alta vulnerabilidad. Todo lo anterior conllevará consecuencias importantes dentro del orden económico y social, con afectaciones psicológicas y de salud para la población.

\section{Conclusiones}

A través de la línea discursiva expuesta se ha intentado ofrecer un trazo de acción de cómo a través del proceso de gestión del riesgo, y particularmente de uno de sus componentes, la vulnerabilidad, se puede dar respuesta organizadamente para atenuar las intensidades de las sequías e inundaciones en México, debido la imposibilidad de controlar y disminuir el potencial existente por el peligro. La gestión del riesgo en el caso de sequías, así como en el de inundaciones, hasta ahora ha conllevado una respuesta pública sobre todo a partir de las situaciones extremas, para mitigar las alteraciones, y es esta condición la que demarca lo que se hace y no se hace en el futuro.

Rara vez se ha contemplado la relación existente en estos escenarios entre el ambiente, el desarrollo y los desastres, con una visión de carácter multidimensional que lleve a una reducción del riesgo, con la finalidad de prevenir, mitigar y preparase, en el marco del desarrollo sustentable, a los impactos adversos de los peligros que inciden en la intensidad del riesgo. Por lo tanto, desde este marco de referencia, una adecuada gestión del riesgo se constituye en un instrumento para establecer los planes de ordenamiento territorial, a fin de evitar la construcción de escenarios presentes y futuros que incrementen la vulnerabilidad y agraven los efectos de las amenazas (Merayo Rodríguez y Berzaga Sablón, 2010).

De acuerdo con Pauwels et al. (2011),

Todo sistema territorial contiene una amplia y compleja información aportada por sus recursos naturales, su estructura geomorfológica y las comunidades o grupos humanos que lo ocupan, que reconocemos como las grandes dimensiones de un proceso integral de construcción, intervención, habilitación y proyección del territorio. (p. 51)

Es así como debe encausarse el esfuerzo gubernamental y social, a partir de acciones planeadas con mecanismos compensatorios o protectores que provean opciones de reducir la vulnerabilidad creada por el peligro en el tiempo. En México es palpable que se han realizado esfuerzos, como la Ley General de Protección Civil y el Programa Especial de Cambio Climático, para impulsar una cultura de prevención y precautoria, especialmente en el marco de la aplicabilidad de las políticas públicas, cuyo manejo se había alejado del contexto de la gestión del riesgo y se reflejó en la toma de decisiones sin una percepción social del riesgo, lo cual ha traído incertidumbres normativas e institucionales 
en el concierto nacional que hoy se desea subsanar (Neri y Briones, 2012). Finalmente, como afirma Moreno Plata (2011), una adecuada gestión del riesgo se debe supeditar a un "razonamiento de carácter hipotético [precautorio], lo que puede posibilitar la reducción de las principales incertidumbres en el plano normativo, cognitivo o institucional” (p. 155).

\section{Referencias}

Alpízar Marín, M. L. (2009). Educación y reducción de riesgos y desastres en Centroamérica: Gestión del riesgo. San José: Coordinación Educativa y Cultural Centroamericana.

Álvarez-Gordillo, G. C., Álvarez-Gordillo, L. M., Eroza-Solana, E. y Dorantes-Jiménez, J. E. (2008). Propuesta educativa para la gestión del riesgo de desastres en la región sierra de Chiapas, México. Revista Mexicana de Investigación Educativa, 13(38), 919-943.

Angelotti Pasteur, G. (2014). Acciones gubernamentales frente a los desastres provocados por fenómenos hidrometeorológicos en México. Gestión y Ambiente, 17(2), 69-83.

Audefroy, J. F. (2012). Adaptación de la vivienda vernácula a los climas en México. En V. García Acosta, J. F. Audefroy y F. Briones (Coords.), Estrategias sociales de prevención y adaptación (pp. 95-106). México: Centro de Investigaciones y Estudios Superiores en Antropología Social.

Barkin, D. (2006). La gestión del agua urbana en México: Retos, debates y bienestar. México: Universidad de Guadalajara.

Cantú-Martínez, P. C. (2012). El conflicto ambiental del agua en los albores del siglo XXI. Ciencia UANL, 15(59), 20-29.

Cantú-Martínez, P. C. (2014). Cambio climático: sus repercusiones para la sustentabilidad. Ciencia UANL, 17(67), $31-36$.

Carabias, J. y Landa, R. (2005). Agua, medio ambiente y sociedad: Hacia la gestión integral de los recursos hidricos en México. México: Universidad Nacional Autónoma de México/Colegio de México/Fundación Gonzalo Río Arronte.

Casillas González, J. A. (2004). La visión de la Sagarpa para el desarrollo integral de microcuencas hidrográficas. En H. Cotler (Comp.), El manejo integral de cuencas en México: estudios y reflexiones para orientar la política ambiental (pp. 211-221). México: Secretaría de Medio Ambiente y Recursos Naturales-Instituto Nacional de Ecología.

Castorena, G., Sánchez, E., Florescano, E., Padilla, G. y Rodríguez, L. (1980). Análisis histórico de las sequias en México. México: Comisión del Plan Nacional Hidráulico, Secretaría de Agricultura y Recursos Hidráulicos.

Centro Nacional de Prevención de Desastres. (2001). Serie Impacto socioeconómico de los desastres naturales 2000.

México: Centro Nacional de Prevención de Desastres-SEGOB.

Comisión Intersecretarial de Cambio Climático. (2012). Informe de Avances del Programa Especial de Cambio Climático 2009-2012. México: Secretaría de Medio Ambiente y Recursos Naturales.

Comisión Intersecretarial de Cambio Climático. (2014). Versión de difusión del Programa Especial de Cambio Climático 2014-2018 (PECC 2014-2018). México: Secretaría de Medio Ambiente y Recursos Naturales. Comisión Nacional del Agua. (2004). Estadísticas del agua en México. México: Autor.

Comisión Nacional del Agua. (2007). Estadísticas del agua en México. México: Conagua/Semarnat. 
Comisión Nacional de Agua. (2013). Estadísticas del Agua en México. México: Semernat-Conagua.

Contreras Servín, C. (2005). Las sequías en México durante el siglo XIX. Investigaciones Geográficas, 56, 118-133.

Fondo para la Comunicación y la Educación Ambiental, Centro Mexicano de Derecho Ambiental y Presencia Ciudadana Mexicana (2006). El agua en México: Lo que todas y todos debemos saber. México: FEACemda-PCM.

García Jiménez, F., Fuentes Mariles, O. y Matías Ramírez, L. G. (2002). Sequías. México: Centro Nacional de Prevención de Desastres-Secretaría de Gobernación.

Grupo Achipáhuac. (2008). El presente apocalíptico: buscando agua limpia en el siglo XXI. En J. Peńa Rodríguez (Coord.), El agua, espejo de los pueblos (pp. 61-82). México: Plaza y Valdes y FES Acatlán.

Keipi, K., Mora Castro, S. y Bastidas, P. (s. f.) La gestión del riesgo dentro del ciclo de los proyectos. Lista de preguntas indicativas ("checklist”). Washington, D. C.: Banco Interamericano de Desarrollo.

Lampis, A. y Rubiano, L. (2012). ¡Y siguen culpando a la lluvia! Vulnerabilidad ambiental y social en el sector Altos de la Estancia, Bogotá, Colombia. En F. Briones (Coord.), Perspectivas de investigación y acción frente al cambio climático en Latinoamérica (pp. 177-219). Caracas: Red de Estudios Sociales en Prevención de Desastres en América Latina.

Ley de Aguas Nacionales. (2013). Diario Oficial de la Federación, Estados Unidos Mexicanos.

Marcos Valiente, O. (2001). Sequía: definiciones, tipologías y métodos de cuantificación. Investigaciones Geográficas, (26), 59-80.

Martínez Austria, P. F. (2001). Paradigmas emergentes para el manejo del agua en el siglo XXI. Ingeniería Hidráulica en México, 16(4), 127-143.

Merayo Rodríguez, A. y Berzaga Sablón, O. S. (2010). El perfeccionamiento de la gestión ambiental desde el análisis de riesgo para la toma eficiente de decisiones. Ciencias Holguín, 16(2), 1-11.

Miguel Velasco, A. E., Maldonado Cruz, P., Torres Valdez, J. C. y Santiago Luis, L. M. (2012). Los efectos del cambio climático en el desarrollo regional. Revista Desarrollo Local Sostenible, 5(15), 1-15.

Monforte García, G. y Cantú-Martínez, P. C. (2009). Escenario del agua en México. CULCyT Cultura Cientifica y Tecnológica, (30), 31-40.

Moreno Plata, M. (2011). El agotamiento del paradigma burocrático ante el riesgo ambiental contemporáneo. Politica y Cultura, (36), 127-155.

Moreno Vázquez, J. L., Marañón Pimentel, B. y López Córdova, D. (2010). Los acuíferos sobreexplotados: Origen, crisis y gestión social. En B. Jiménez Cisneros, M. L. Torregrosa y Armentia y L. Aboites Aguilar (Eds.), El agua en México: Cauces y encauces (pp. 79-116). México: Academia Mexicana de Ciencias/ Comisión Nacional del Agua.

Narváez, L., Lavell, A. y Pérez Ortega, G. (2009). La gestión del riesgo de desastres: Un enfoque basado en procesos. Lima: Comisión Europea/Secretaría General de la Comunidad Andina/Comité Andino para la Prevención y Atención de Desastres (Caprade).

Neri, C. y Briones, F. (2012). Cada quien su sequía. Caracterización de la vulnerabilidad en Sonora, México. En F. Briones (Coord.), Perspectivas de investigación y acción frente al cambio climático en Latinoamérica (pp. 119-150). Caracas: Red de Estudios Sociales en Prevención de Desastres en América Latina.

Oficina del Alto Comisionado de las Naciones Unidas para los Derechos Humanos. (2011). El derecho al agua. Génova: Naciones Unidas. 
Pauwels, V., Pla, I., Beethoven Zuleta, F., Arias, A., Loaiza, J. C., Zapata, R. y Botero, L. (2011). Seminario internacional de prevención de desastres propuesta tecnológica para una atención política integral de la gestión riesgo. Revista Gestión y Ambiente, 14(3), 51-52.

Peña Ramírez, J. (2012). Crisis del agua en Monterrey, Guadalajara, San Luis Potosí, León y la Ciudad de México (1950-2010). México: UNAM.

Peña Ramírez, J. y Grupo Achipáhuac. (2008). Algunas dificultades históricas y cotidianas para resolver los conflictos por el agua en la cuenca de México. En J. Peña Ramírez. (Coord.), El agua espejo de los pueblos. Ensayos de ecología política sobre la crisis del agua en México en el umbral del milenio (pp. 107-130). México: FES Acatlán y Plaza y Valdés Editores.

Pineda Pablos, N., Salazar Adams, A. y Buenfil Rodríguez, M. (2010). Para dar de beber a las ciudades mexicanas: El reto de la gestión eficiente del agua ante el crecimiento urbano. En B. Jiménez Cisneros, M. L. Torregrosa y Armentia y L. Aboites Aguilar (Eds.), El agua en México: Cauces y encauces (pp. 117-140). México: Academia Mexicana de Ciencias/Comisión Nacional del Agua.

Ponce-Cruz, Y. Y., Cantú-Martínez, P. C. y Puente-Quintanilla, J. C. (2013). La gestión ambiental del cambio climático. CULCYT Cultura Cientifica y Tecnológica, 10(51): 14-41.

Rolland, L. y Vega Cárdenas, Y. (2010). La gestión del agua en México. Polis, 6(2), 155-188.

Romero, G. y Maskrey, A. (1993). Como entender los desastres naturales En A. Maskrey (Comp.), Los desastres no son naturales (pp. 6-10). Ciudad de Panamá: Red de Estudios Sociales en Prevención de Desastres en América Latina.

Salas Salinas, M. A. y Jiménez Espinoza, M. (2004). Inundaciones. México: Centro Nacional de Prevención de Desastres-Secretaría de Gobernación.

Secretaría de Gobernación en México. (2008). Tabasco: Características e impacto socioeconómico de las inundaciones provocadas a finales de octubre y a comienzos de noviembre de 2007 por el frente frío número 4 . Resumen. México: Segob-Cepal-Cenapred-Gobierno del Estado de Tabasco.

Secretaría de Gobernación. (2012). Decreto por el que se expide la Ley General de Protección Civily de su Reglamento. Diario Oficial de la Federación.

Secretaría de Medio Ambiente y Recursos Naturales. (2013a). Informe de la situación del medio ambiente en México. Compendio de estadísticas ambientales. Indicadores clave y de desempeño ambiental. Edición 2012. México: Secretaría de Medio Ambiente y Recursos Naturales.

Secretaría de Medio Ambiente y Recursos Naturales. (2013b). Análisis espacial de las regiones más vulnerables ante las sequías en México. México: Semarnat-Gonagua.

Toscana Aparicio, A. (2011). Protección civil, población, vulnerabilidad y riesgo en Santiago Miltepec, Toluca. Investigaciones Geográficas, (74), 35-47.

Velasco, I., Ochoa, L. y Gutiérrez, C. (2005). Sequía, un problema de perspectiva y gestión. Región y Sociedad, $17(34), 35-71$.

Velázquez Simental, L. del C. (2012). El proceso de desarrollo del sistema nacional de información del agua en México (SINA). Avances, limitantes y retos (tesis de maestría). Universidad Nacional Autónoma de México, Ciudad de México.

Wilches-Chaux, G. (1993). La vulnerabilidad global En A. Maskrey (Comp.), Los desastres no son naturales. (pp. 11-44). Ciudad de Panamá: Red de Estudios Sociales en Prevención de Desastres en América Latina. 